\title{
Systematic Review \\ The Usefulness of Serological Inflammatory Markers in Patients with Rotator Cuff Disease-A Systematic Review
}

\author{
Chi Ngai Lo ${ }^{1, * \mathbb{C}}$, Bernard Pui Lam Leung ${ }^{2}$ and Shirley Pui Ching Ngai ${ }^{3}$ \\ 1 Family Care Physiotherapy Clinic, 612 Clementi West St. 1, Singapore 120612, Singapore \\ 2 Health and Social Sciences Cluster, Singapore Institute of Technology, 10 Dover Drive, Singapore 138683, \\ Singapore; bernard.leung@singaporetech.edu.sg \\ 3 Department of Rehabilitation Sciences, The Hong Kong Polytechnic University, Hong Kong SAR, China; \\ shirley.ngai@polyu.edu.hk \\ * Correspondence: lochingai@gmail.com
}

Citation: Lo, C.N.; Leung, B.P.L.; Ngai, S.P.C. The Usefulness of Serological Inflammatory Markers in Patients with Rotator Cuff Disease-A Systematic Review. Medicina 2022, 58, 301. https:// doi.org/10.3390/medicina58020301

Academic Editor: Vassilios S. Nikolaou

Received: 9 December 2021 Accepted: 13 February 2022 Published: 16 February 2022

Publisher's Note: MDPI stays neutral with regard to jurisdictional claims in published maps and institutional affiliations.

Copyright: (C) 2022 by the authors. Licensee MDPI, Basel, Switzerland. This article is an open access article distributed under the terms and conditions of the Creative Commons Attribution (CC BY) license (https:// creativecommons.org/licenses/by/ $4.0 /)$.

\begin{abstract}
Background and Objectives: Rotator cuff disease (RCD) is a prominent musculoskeletal pain condition that spans a variety of pathologies. The etiology and precise diagnostic criteria of this condition remain unclear. The current practice of investigating the biochemical status of RCD is by conducting biopsy studies but their invasiveness is a major limitation. Recent biochemical studies on RCD demonstrate the potential application of serological tests for evaluating the disease which may benefit future clinical applications and research. This systematic review is to summarize the results of available studies on serological biochemical investigations in patients with RCD. Methods: An electronic search on databases PubMed and Virtual Health Library was conducted from inception to 1 September 2021. The inclusion criteria were case-control, cross-sectional, and cohort studies with serological biochemical investigations on humans with RCD. Methodological quality was assessed using the Study Quality Assessment Tool for Observational Cohort and Cross-sectional studies from the National Heart, Lung, and Blood Institute. Results: A total of 6008 records were found in the databases; of these, 163 full-text studies were checked for inclusion and exclusion criteria. Nine eligible studies involving 984 subjects with RCD emerged from this systematic review. The quality of the studies found ranged from poor to moderate. In summarizing all the studies, several fatty acids, nonprotein nitrogen, interleukin- $\beta$, interleukin- 8 , and vascular endothelial growth factor were found to be significantly higher in blood samples of patients with RCD than with control group patients, while Omega-3 Intex, vitamin B12, vitamin D, phosphorus, interleukin-10, and angiogenin were observed to be significantly lower. Conclusions: This is the first systematic review to summarize current serological studies in patients with RCD. Results of the studies reflect several systemic physiological changes in patients with RCD, which may prove helpful to better understand the complex pathology of RCD. In addition, the results also indicate the possibility of using serological tests in order to evaluate RCD; however, further longitudinal studies are required.
\end{abstract}

Keywords: rotator cuff disease; biomarkers; blood test; systematic review

\section{Introduction}

Rotator cuff disease (RCD) spans a variety of pathologies including rotator cuff (RC) tendon impingement in the subacromial space, partial tendon tears, full-thickness tears, and cuff tear arthropathy [1]. RCD is a prominent condition in musculoskeletal pain, with a prevalence reported to be between $18 \%$ and $31 \%$ in the general population, and in over $66.7 \%$ of people throughout their lifetimes [2]. Moreover, the incidence rate of surgical intervention for RCD has been significantly increasing in the US and Australia $[3,4]$.

The etiology and precise diagnostic criteria of this condition are still unclear. Recently, multiple studies have observed significantly elevated levels of pro-inflammatory mediators in biopsies of rotator cuff tendons in patients with $\operatorname{RCD}[5,6]$, indicating that inflammatory 
processes may play a significant role in the development of disease [7]. These findings are helpful to explore underlying pathologies and potential diagnostic tools. Recent research evidence demonstrates an association between RCD and metabolic syndrome [8]. However, the invasiveness of biopsy studies in RCD remains a major limitation as biopsies hinder the availability of repetitive measures in longitudinal biochemical studies and in clinical disease monitoring.

Serological investigation is a more convenient, lower cost, less time-consuming, and less invasive means to evaluate systemic bio-physiological changes. Increasing evidence supports serological investigation for RCD, and raises the prospect of using serological biomarkers in monitoring the responsiveness of RCD to current treatments. At present, it remains unclear if any specific biomarker can reflect disease status, severity, and clinical implications. The aim of this systematic review is to summarize recent findings from available serological biochemical studies in patients with RCD compared to people without shoulder pathologies.

\section{Materials and Methods}

\subsection{Study Design and Registration}

This systematic review was conducted following procedures in the Cochrane Handbook [9], and the review is reported according to the Preferred Reporting Items for Systematic Review and Meta-Analysis (PRISMA) guidelines [10]. The protocol was registered on the International Prospective Register of Systematic Reviews (PROSPERO), CRD42021224718.

\subsection{Search Strategy}

To summarize and update available reports on physiological changes in patients with RCD, a literature search using electronic search engines PubMed (including MEDLINE and PubMed Central) and Virtual Health Library (including MEDLINE, Latin America and Caribbean Literature in Health Sciences LILACS, Índice Bibliográfico Espanhol de Ciências da Saúde IBECS, and BENF Nursing) was conducted from inception to 1 September 2021. The language of articles chosen was limited to English only. The keywords and search strategy used are detailed in Appendices A.1 and A.2. The reference lists of the included articles in addition to Google Scholar were checked for additional eligible articles.

\subsection{Eligibility Criteria}

- Student Design-inclusion criteria were case-control studies, cross-sectional studies, or cohort studies.

- Population - subjects of the studies comprised adult ( $>18$ years old) humans with RCD, including diagnosis of RC tendinopathy, shoulder impingement, subacromial bursitis, and RC tears. The diagnoses of RCD were confirmed by radiological or surgical examinations. Study populations involving other shoulder pathologies such as fractures, frozen shoulders, and shoulder joint dislocations were excluded. Subjects with other systemic conditions or comorbidities which could affect the outcomes were excluded. In addition, the studies were accompanied by suitable control groups without pathologies in the shoulders or without systemic conditions such as inflammatory joint disease and without immunological or neoplastic disorders. All animal studies were excluded.

- Outcomes-the studies needed to have outcome measures that could objectively quantify physiological biomarkers in blood samples of the subjects. Studies that lacked statistical analyses to compare their outcomes with were excluded.

\subsection{Study Selection}

Search records were imported into the referencing website EndNote Web (https:/ / access.clarivate.com, accessed on 6 September 2021) for duplication elimination. Based on the inclusion criteria, abstracts and titles were primarily screened by two independent 
reviewers (CL and BL), followed by screening of the full-text articles. A third reviewer (SN) resolved disagreements. Reviewer CL is a physiotherapist with more than 15 years of clinical experience, a masters degree with research training, and more than 10 years of experience in musculoskeletal clinical research. Reviewer BL is a researcher with a $\mathrm{PhD}$ degree in immunology and more than 25 years of research experience in biomarkers and inflammatory diseases. Reviewer $\mathrm{SN}$ is a researcher with a PhD degree in physiotherapy with specialized training in epidemiology and biostatistics, plus more than 15 years of research experience in exercise physiology. Manual searching of included studies' reference lists was performed to identify additional eligible studies. Meta-analysis was planned to determine if selected articles have comparable outcome measures.

\subsection{Quality and Risk of Bias Assessment}

Selected articles were appraised on quality using the Study Quality Assessment Tool for Observational Cohort and Cross-sectional studies from the National Heart, Lung, and Blood Institute (https:/ / www.nhlbi.nih.gov/health-topics/study-quality-assessmenttools, accessed on 30 September 2021). This assessment tool consists of 14 questions to evaluate each study's internal validity and risks of selection bias, information bias, measurement bias, or confounding bias. Quality and risk of bias assessment were conducted by two reviewers (CL and BL) individually. Any inconsistent results were discussed and resolved together with the third reviewer (SN).

\subsection{Data Extraction}

Data were extracted from selected papers by one reviewer (CL) and checked by a second reviewer (SN) using a piloted table designed by the authors. The data included study design, demographics of the subjects, sample size, diagnosis for RCD, types of serological measures, results, and statistical analysis. If data in a selected study were missing or not clear, its authors were contacted to request details. The primary focus of the results was on identifying differences in the average levels of serological biomarkers between study groups and control groups in addition to statistical significances, if any.

\section{Results}

The process flow of initial search results, screening, inclusion, and exclusion of full texts is illustrated in Figure 1. A total of 6008 records were found in the selected databases. After the removal of duplicates, 3470 records were screened using an automated filter for English, human subjects, study designs, along with reviewers screening for titles and abstracts. A total of 163 studies were checked through full-text screening; of those, 152 studies were excluded since their study designs or study populations did not match the selection criteria. Two more studies $[11,12]$ were excluded during data extraction since their outcome measures are genetic factors rather than biochemical substances. Ultimately nine studies were selected from this systematic review.

\subsection{Study Characteristics}

Characteristics of the included studies are presented in Table 1. These articles comprised eight case-control studies and one cohort study. Most of the population in the experimental group were patients with RC tears confirmed by MRI or surgical investigation and clinical tests but Sayitskaya et al.'s (2011) study also included patients with RC inflammation and tendon degeneration [13]. From the included studies, a total of 984 patients with RCD comprised the experimental group. The control group's subjects were similar in gender ratio and age with those of the experimental group but without evidence of shoulder pathologies or conditions that could have affected the serological tests. Serological assessments were conducted through plasma or serum samples in order to measure levels of matrix metalloproteinase (MMP), tissue inhibitors of metalloproteinases (TIMPs), fatty acids, Omega-3 index, cholesterols, fasting plasma glucose, triglyceride, 
fibrinogen, nonprotein nitrogen (NPN), creatinine, interleukins (ILs), vascular endothelial growth factor (VEGF), angiogenin (ANG), vitamin D, vitamin B12, and other substances.

Figure 1 Flowchart of the literature search.

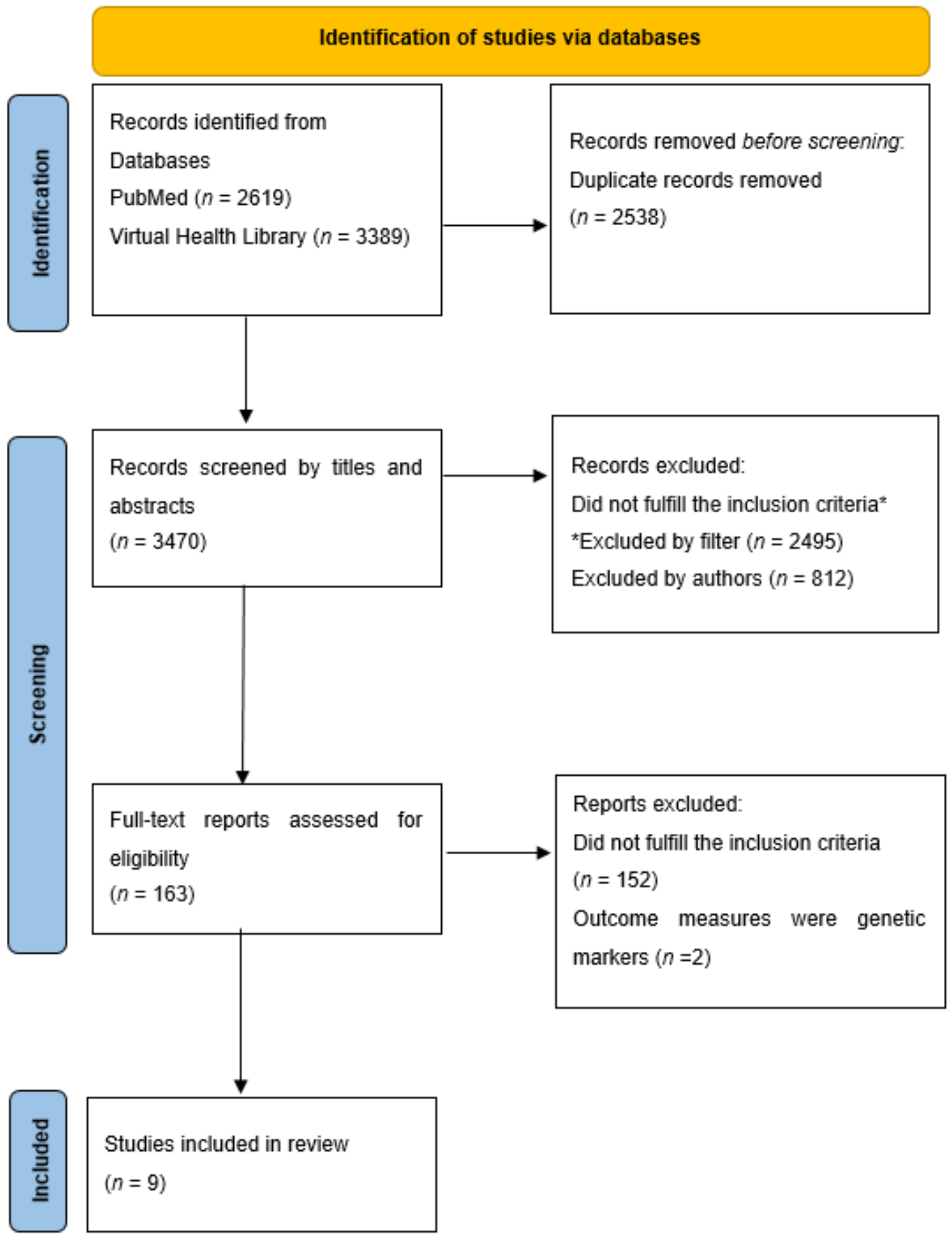

Figure 1. Diagnosis of RCD was confirmed by radiological or surgical examination. Exclusion criteria-study populations involving other shoulder pathologies such as fractures, frozen shoulders, and shoulder joint dislocations were excluded. Subjects with other systemic conditions or comorbidities which could affect the outcomes were excluded. In addition, the studies were accompanied by suitable control groups without pathologies in the shoulders or systemic conditions such as inflammatory joint disease, immunological, or neoplastic disorders. All animal studies were excluded. (* Filter by English language, human subjects, and study designs). 
Table 1. Summary of study characteristics and results of included studies.

\begin{tabular}{|c|c|c|c|c|}
\hline Author & $\begin{array}{l}\text { Study Design and } \\
\text { Level of Evidence }\end{array}$ & Population and Diagnosis & Biomarkers & Results \\
\hline $\begin{array}{l}\text { Hallgren et al., } \\
2012\end{array}$ & $\begin{array}{l}\text { Case-Control } \\
\text { Level III }\end{array}$ & $\begin{array}{l}\text { Experimental group } \\
17 \text { patients with sonographically } \\
\text { verified rotator cuff tears } \\
\text { Mean age } 61 \text { (39-77) years } \\
\text { Control group } \\
\# 16 \text { age and sex-matched individuals } \\
\text { with no history of shoulder disease and } \\
\text { sonographically intact rotator cuffs }\end{array}$ & $\begin{array}{l}\text { Plasma samples } \\
\text { MMP-1 } \\
\text { MMP-2 } \\
\text { MMP-3 } \\
\text { MMP-7 } \\
\text { MMP-9 } \\
\text { TIMP-1 } \\
\text { TIMP-2 } \\
\text { TIMP-3 } \\
\text { TIMP-4 }\end{array}$ & $\begin{array}{l}\text { TIMP-1, median (range) } \\
\text { Experimental group } \\
86(67-119) \mathrm{ng} / \mathrm{mL} \\
\text { Control group } \\
78(66-93) \mathrm{ng} / \mathrm{mL} \\
\text { Measured by Luminex, } p=0.04 \\
\text { Not significant when repeated using ELISA } \\
\text { method, } p=0.2 \\
\text { Other measures not significant }\end{array}$ \\
\hline Hudek et al., 2019 & $\begin{array}{l}\text { Case-Control } \\
\text { Level III }\end{array}$ & $\begin{array}{l}\text { Experimental group } \\
29 \text { patients with non-traumatic } \\
\text { complete rotator-cuff tears } \\
\text { ( } 22 \text { males, } 7 \text { females; mean age: } 53.9 \pm \\
7.1 \text { years) } \\
\text { Diagnosis confirmed by MRI and } \\
\text { surgery } \\
\text { Control group } \\
15 \text { non-smoking subjects with healthy } \\
\text { shoulders } \\
\text { ( } 10 \text { males, } 5 \text { females; mean age } 52.5 \pm \\
6.5 \text { years) }\end{array}$ & $\begin{array}{l}\text { Plasma sample } \\
\text { Fatty acids } \\
\text { C16:1n-7 } \\
\text { C20:2n-6 } \\
\text { C24:0 } \\
\text { C24:1n-9 } \\
\text { C22:6n-3 } \\
\text { Omega-3 Index }\end{array}$ & $\begin{array}{l}\text { C16:1n-7 } \\
\text { Exp: } 0.59 \pm 0.29 \\
\text { Control: } 0.33 \pm 0.12 \\
{ }^{*} p<0.01 \\
\text { C20:2n-6 } \\
\text { Exp: } 0.22 \pm 0.04 \\
\text { Control: } 0.19 \pm 0.02 \\
{ }^{*} p=0.02 \\
\text { C24:0 } \\
\text { Exp: } 0.71 \pm 0.24 \\
\text { Control: } 0.52 \pm 0.21 \\
{ }^{*} p=0.01 \\
\text { C24:1n-9 } \\
\text { Exp: } 0.78 \pm 0.17 \\
\text { Control: } 0.65 \pm 0.20 \\
{ }^{*} p=0.04 \\
\text { C22:6n-3 } \\
\text { Exp: } 4.23 \pm 1.11 \\
\text { Control: } 5.10 \pm 1.12 \\
{ }^{*} p=0.02 \\
\text { Omega-3 Index } \\
\text { Exp: } 5.01 \pm 1.27 \\
\text { Control: } 6.01 \pm 1.39 \\
{ }^{*} p=0.03\end{array}$ \\
\hline Park et al., 2018 & $\begin{array}{l}\text { Cohort study } \\
\text { Level III }\end{array}$ & $\begin{array}{l}\text { Experiment group } \\
199 \text { patients with posterosuperior } \\
\text { rotator cuff tears confirmed by MRI } \\
143 \text { patients were symptomatic } \\
107 \text { male }(53.8 \%) \\
\text { Age } 61.9 \pm 7.6 \\
\\
\text { Control group } \\
435 \text { individuals with intact RC } \\
200 \text { male }(46.0 \%) \\
\text { Age } 57.7 \pm 8.8\end{array}$ & $\begin{array}{l}\text { Serum lipid levels } \\
\text { Total cholesterol } \\
\text { LDL } \\
\text { TG } \\
\text { HDL } \\
\text { Non-HDL }\end{array}$ & $\begin{array}{l}\text { Experimental group } \\
\text { Total cholesterol } 200 \pm 35 \\
\text { LDL } 133 \pm 33 \\
\text { TG } 109(83-60) \\
\text { HDL } 54(45-63) \\
\text { Non-HDL } 145 \pm 35 \\
\text { Control group } \\
\text { Total cholesterol } 198 \pm 37 \\
\text { LDL } 131 \pm 34 \\
\text { TG } 104(78-150) \\
\text { HDL } 57(46-67) \\
\text { Non-HDL } 140 \pm 37 \\
\text { Unit: mg } / \text { dL } \\
\text { HDL odds ratio }=0.99(0.98-1.00), p=0.035 \\
\text { Other measures not significant } \\
\text { Dyslipidemia-Hypo-HDLemia } \\
\text { Experimental group }=62(31.2 \%) \\
\text { Control group }=75(17.2 \%) \\
\text { Hypo-HDLemia odds ratio }=2.17 \\
(1.47-3.21),{ }^{*} p<0.001\end{array}$ \\
\hline
\end{tabular}


Table 1. Cont.

\begin{tabular}{|c|c|c|c|c|}
\hline Author & $\begin{array}{l}\text { Study Design and } \\
\text { Level of Evidence }\end{array}$ & Population and Diagnosis & Biomarkers & Results \\
\hline Longo et al., 2009 & $\begin{array}{l}\text { Case-control } \\
\text { Level III }\end{array}$ & $\begin{array}{l}\text { Experimental group } \\
97 \text { patients with rotator cuff tears } \\
\text { ( } 36 \text { men and } 61 \text { women; mean age: } \\
62.9 \text { years, range } 37 \text { to } 82 \text { ) } \\
\text { Diagnosis was based on clinical and } \\
\text { imaging grounds and surgery } \\
\text { Control group } \\
97 \text { patients with no evidence of } \\
\text { shoulder pathologies } \\
(36 \text { men and } 61 \text { women; mean age: } \\
61.6 \text { years, range } 36 \text { to } 80 \text { ) }\end{array}$ & $\begin{array}{l}\text { Venous fasting plasma } \\
\text { glucose levels }\end{array}$ & $\begin{array}{l}\text { Experimental group } \\
99.17 \pm 9.04 \mathrm{mg} \text { per decilitre } \\
5.5 \pm 0.5 \text { millimoles per litre } \\
\text { Control group } \\
95.45 \pm 9.87 \mathrm{mg} \text { per decilitre } \\
5.3 \pm 0.55 \text { millimoles per litre } \\
* p<0.01 \text {, both groups were within the } \\
\text { normoglycemic range }\end{array}$ \\
\hline Longo et al., 2010 & $\begin{array}{l}\text { Case-control } \\
\text { Level III }\end{array}$ & $\begin{array}{l}\text { Experimental group } \\
120 \text { patients underwent arthroscopic } \\
\text { repair of rotator cuff tears } \\
\text { (45 men and } 75 \text { women; mean age: } \\
64.86 \text { years) } \\
\text { Diagnosis was confirmed by MRI } \\
\text { Control group } \\
120 \text { patients underwent knee } \\
\text { arthroscopic meniscectomies with no } \\
\text { evidence of shoulder pathologies } \\
\text { (45 men and } 75 \text { women; mean age: } \\
63.91 \text { years) }\end{array}$ & $\begin{array}{l}\text { Serum triglyceride and } \\
\text { total cholesterol } \\
\text { concentrations }\end{array}$ & $\begin{array}{l}\text { Serum triglycerides } \\
\text { Unit in mg per decilitre and millimoles per } \\
\text { litre } \\
\text { Experimental group } \\
\text { Male } \\
158.42 \pm 122.29 \\
1.81 \pm 1.39 \\
\text { Female } \\
131.81 \pm 55.9 \\
1.49 \pm 0.63 \\
\text { Control group } \\
\text { Male } \\
139.87 \pm 75.56 \\
1.58 \pm 0.85 \\
\text { Female } \\
120.48 \pm 53.75 \\
1.36 \pm 0.61 \\
\text { Serum cholesterol } \\
\text { Unit in mg per decilitre and millimoles per } \\
\text { litre } \\
\text { Experimental group } \\
\text { Male } \\
212.76 \pm 40.58 \\
5.51 \pm 1.05 \\
\text { Female } \\
224.11 \pm 44.42 \\
5.80 \pm 1.15 \\
\text { Control group } \\
\text { Male } \\
213.6 \pm 36.45 \\
5.53 \pm 0.94 \\
\text { Female } \\
217.3 \pm 39.28 \\
5.63 \pm 1.02 \\
\text { not statistically significant differences either } \\
\text { in triglyceride concentration }(p=0.6) \text { or } \\
\text { total cholesterol concentration }(p=0.1) \\
\end{array}$ \\
\hline
\end{tabular}


Table 1. Cont.

\begin{tabular}{|c|c|c|c|c|}
\hline Author & $\begin{array}{l}\text { Study Design and } \\
\text { Level of Evidence }\end{array}$ & Population and Diagnosis & Biomarkers & Results \\
\hline Longo et al., 2014 & $\begin{array}{l}\text { Case-control } \\
\text { Level III }\end{array}$ & $\begin{array}{l}\text { Experimental group } \\
82 \text { patients underwent arthroscopic } \\
\text { repair of RC tears } \\
\text { ( } 36 \text { men and } 46 \text { women; mean age: } 57.7 \\
\pm 10.2 \text { years) } \\
\text { Diagnosis confirmed by MRI } \\
\text { Control group } \\
82 \text { patients underwent arthroscopic } \\
\text { meniscectomies for meniscal tears with } \\
\text { no history of RC symptoms } \\
\text { ( } 36 \text { men and } 46 \text { women; mean age: } 55.9 \\
\pm 9 \text { years) }\end{array}$ & $\begin{array}{l}\text { Serum fibrinogen } \\
\text { concentration. }\end{array}$ & $\begin{array}{l}\text { Experimental group } \\
335.9 \pm 171 \mathrm{mg} / \mathrm{dL} \text { (range } 70-512 ; \text { median } \\
328.5) \\
\text { Control group } \\
329.6 \pm 205 \mathrm{mg} / \mathrm{dL} \text { (range } 72-607 \text {; median } \\
322.5 \text { ) } \\
p=0.05\end{array}$ \\
\hline Papalia et al., 2011 & $\begin{array}{l}\text { Case-control } \\
\text { Level III }\end{array}$ & $\begin{array}{l}\text { Experimental group } \\
200 \text { patients who underwent } \\
\text { arthroscopic repair of rotator cuff tears } \\
\text { ( } 93 \text { men, } 107 \text { women; age } 56.8 \\
\pm 11.7 \text { years) } \\
\text { Diagnosis was confirmed by } \\
\text { preoperative imaging and arthroscopy } \\
\text { Control group } \\
200 \text { patients who underwent knee } \\
\text { arthroscopies for management of } \\
\text { meniscal tears, with or without } \\
\text { cartilage damage } \\
\text { (93 men and } 107 \text { women, age of } 53.9 \pm \\
12.6 \text { years) }\end{array}$ & $\begin{array}{l}\text { Plasma Nonprotein } \\
\text { nitrogen (NPN) and } \\
\text { creatinine levels }\end{array}$ & $\begin{array}{l}\text { NPN } \\
\text { Experimental group } \\
37.2 \pm 8.9 \mathrm{mg} / \mathrm{dL} \\
2.06 \pm 0.49 \mathrm{mmol} / \mathrm{L} \\
\text { Control group } \\
35.9 \pm 10.2 \mathrm{mg} / \mathrm{dL} \\
1.98 \pm 0.56 \mathrm{mmol} / \mathrm{L} \\
* p=0.035 \\
\text { Creatinine } \\
\text { Experimental group } \\
0.8 \pm 0.19 \mathrm{mg} / \mathrm{dL} \\
0.04 \pm 0.01 \mathrm{mmol} / \mathrm{L} \\
\text { Control group } \\
0.82 \pm 0.18 \mathrm{mg} / \mathrm{dL} \\
0.045 \pm 0.01 \mathrm{mmol} / \mathrm{L} \\
p=0.66\end{array}$ \\
\hline $\begin{array}{l}\text { Savitskaya et al., } \\
2011\end{array}$ & $\begin{array}{l}\text { Case-control } \\
\text { Level III }\end{array}$ & $\begin{array}{l}\text { Experimental group } \\
200 \text { patients with significant } \\
\text { inflammation, tendon degeneration, } \\
\text { and partial or full-thickness rotator cuff } \\
\text { tears } \\
\text { (112 males, } 88 \text { females, age } 40.3 \pm 10.9) \\
\text { Diagnosis was confirmed by MRI } \\
\text { Control group } \\
200 \text { age and sex matched healthy } \\
\text { individuals with no medical history of } \\
\text { rotator cuff disease } \\
\text { (107 males, } 93 \text { females, age } 43.3 \pm 11.5)\end{array}$ & $\begin{array}{l}\text { Serum samples } \\
\text { IL-1 } \beta \\
\text { IL-8 } \\
\text { IL-10 } \\
\text { VEGF } \\
\text { ANG }\end{array}$ & $\begin{array}{l}\text { Experimental group } \\
\text { IL-1 } 16.17 \pm 6.71-43.71 \pm 8.91 \\
\text { IL- } 15.31 \pm 0.85-27.81 \pm 1.11 \\
\text { IL-10 } 3.11 \pm 1.91-7.64 \pm 1.11 \\
\text { VGEF } 402.11 \pm 88.11-621.24 \pm 301.11 \\
\text { ANG } 166.45 \pm 44.90-89.39-40.19 \\
\text { Control group } \\
\text { IL-1 } 3.33 \pm 0.69 \\
\text { IL-8 } 9.11 \pm 0.98 \\
\text { IL-10 } 9.53 \pm 1.21 \\
\text { VEGF } 339.67 \pm 74.65 \\
\text { ANG } 239.51 \pm 58.4 \\
\text { Unit in pg/mL } \\
\text { * IL-1 } \beta, \text { IL-8, and VEGF levels were } \\
\text { significantly higher in RCD patients than in } \\
\text { controls. Serum ANG and IL-10 levels were } \\
\text { significantly lower in RCD patients than in } \\
\text { controls } \\
\text { Exact } p \text { values not provided } \\
\text { * Overexpression of VEGF correlated with } \\
\text { advanced } \\
\text { disease }(\mathrm{r}=0.75 ; p<0.01) \text {, average } \\
\text { microvascular } \\
\text { density ( } \mathrm{r}=0.68, p<0.01) \text {, and visual } \\
\text { analog score } \\
\text { ( } \mathrm{r}=0.75, p<0.01) \text { in patients with RCD. }\end{array}$ \\
\hline
\end{tabular}


Table 1. Cont.

\begin{tabular}{|c|c|c|c|c|}
\hline Author & $\begin{array}{l}\text { Study Design and } \\
\text { Level of Evidence }\end{array}$ & Population and Diagnosis & Biomarkers & Results \\
\hline Kim et al., 2021 & $\begin{array}{l}\text { Case-control } \\
\text { Level III }\end{array}$ & $\begin{array}{l}\text { Experimental group } \\
40 \text { patients with degenerative RC tears } \\
\text { Diagnosis was confirmed by MRI } \\
\text { ( } 23 \text { males, } 17 \text { females, age } 61.0 \pm 5.3) \\
\text { Control group }(n=47) \\
40 \text { patients with minor non-shoulder } \\
\text { trauma but no RC tears or associated } \\
\text { symptoms or clinical signs }\end{array}$ & $\begin{array}{l}\text { Serum sample } \\
\text { Glucose } \\
\text { Magnesium } \\
\text { Calcium } \\
\text { Phosphorus } \\
\text { Zinc } \\
\text { Homocysteine } \\
\text { Vitamin D } \\
\text { Vitamin B12 } \\
\text { Folate }\end{array}$ & $\begin{array}{l}\text { Vitamin B12 } \\
\text { Experimental group } \\
528.4 \pm 145.7 \mathrm{pg} / \mathrm{mL} \\
\text { Control group } \\
627.1 \pm 183.0 \mathrm{pg} / \mathrm{mL} \\
{ }^{*} p=0.007 \\
\text { Vitamin } \mathrm{D} \\
\text { Experimental group } \\
15.7 \pm 7.2 \mathrm{ng} / \mathrm{mL} \\
\text { Control group } \\
21.6 \pm 10.0 \mathrm{ng} / \mathrm{mL} \\
{ }^{*} p=0.002 \\
\text { Vit D odds ratio (OR) for degenerative RC } \\
\text { tear }=0.89 ; 95 \% \mathrm{CI}=0.82-0.96 ;{ }^{*} p=0.006 \\
\text { Phosphorus } \\
\text { Experimental group } \\
3.2 \pm 0.6 \mathrm{mg} / \mathrm{dL} \\
\text { Control group } \\
3.6 \pm 0.7 \mathrm{mg} / \mathrm{dL} \\
{ }^{*} p=0.008 \\
\text { other parameters showed no significant } \\
\text { relationships }\end{array}$ \\
\hline
\end{tabular}

\# No exact data of genders and age were provided. Abbreviations: MMP = matrix metalloproteinase, TIMP = tissue inhibitor of metalloproteinases, IL = interleukin, LDL = low-density lipoprotein, TG = triglyceride, HDL = high-density lipoprotein, hypoHDLemia = hypo-high-density lipoproteinemia, VEGF $=$ Vascular endothelial growth factor, and ANG = angiogenin. ELISA = Enzyme-linked immunosorbent assay. ${ }^{*}$ Results with statistical significance, $p<0.05$.

The levels of fatty acids, nonprotein nitrogen, interleukin-1 $\beta$, IL-8, and VEGF were significantly higher in the blood samples of patients with RCD than those in the control group. In contrast, the levels of Omega-3 Index, vitamin B12, vitamin D, phosphorus, IL-10, and ANG were significantly lower. Although Hallgren et al. (2012) found elevated plasma levels of TIMP-1 in their study group, the result is uncertain because of differences reported between the investigations by Luminex and ELISA methods [14]. Other biomarkers were reported as either not statistically significant or clinically different between experimental and control groups.

\subsection{Quality and Risk of Bias of Selected Articles}

According to the Levels of Evidence for Prognostic Studies, the nine included studies were level III evidence [15]. Assessment of risk of bias and methodologic quality is presented in Table 2. Possible risk of bias could be present as most selected studies do not demonstrate sample size justification. All the studies are cross-sectional and the timeframe is insufficient to support an association between a biochemical substance and RCD. It is possible for the studies to have repetitive measures and longer follow-up periods yet the studies only feature single measurements of serological tests. The selected studies fulfil five to nine out of the fourteen items listed on the assessment tool, with item 8 not being applicable. The quality of the studies is considered poor to fair. 
Table 2. Critical appraisal of included studies. Study Quality Assessment Tool for Observational Cohort and Cross-sectional studies, the National Heart, Lung, and Blood Institute.

\begin{tabular}{|c|c|c|c|c|c|c|c|c|c|}
\hline & \multicolumn{2}{|c|}{$\begin{array}{cc}\text { Hallgren et al., } & \text { Hudek et al., } \\
2012 & 2019\end{array}$} & $\begin{array}{l}\text { Park et al., } \\
\quad 2018\end{array}$ & $\begin{array}{l}\text { Longo et al., } \\
\quad 2009\end{array}$ & $\begin{array}{l}\text { Longo et al., } \\
\quad 2010\end{array}$ & $\begin{array}{l}\text { Longo et al., } \\
\quad 2014\end{array}$ & $\begin{array}{l}\text { Papalia et al., } \\
\quad 2011\end{array}$ & \multicolumn{2}{|c|}{ Savitskaya et al.,Kim et al., } \\
\hline $\begin{array}{l}\text { 1. Was the research question or objective in this } \\
\text { paper clearly stated? }\end{array}$ & $\mathrm{Y}$ & Y & Y & $\mathrm{Y}$ & $\mathrm{Y}$ & $\mathrm{Y}$ & Y & $\mathrm{Y}$ & $\mathrm{Y}$ \\
\hline $\begin{array}{l}\text { 2. Was the study population clearly specified and } \\
\text { defined? }\end{array}$ & $\mathrm{Y}$ & $\mathrm{Y}$ & $\mathrm{N}$ & $\mathrm{Y}$ & $\mathrm{N}$ & $\mathrm{N}$ & $\mathrm{Y}$ & $\mathrm{N}$ & $\mathrm{Y}$ \\
\hline $\begin{array}{l}\text { 3. Was the participation rate of eligible persons at } \\
\text { least } 50 \% \text { ? }\end{array}$ & $\mathrm{Y}$ & Y & Y & Y & Y & Y & Y & Y & Y \\
\hline $\begin{array}{l}\text { 4. Were all the subjects selected or recruited from } \\
\text { the same or similar populations (including the } \\
\text { same time period)? Were inclusion and exclusion } \\
\text { criteria for being in the study prespecified and } \\
\text { applied uniformly to all participants? }\end{array}$ & $\mathrm{Y}$ & $\mathrm{Y}$ & $\mathrm{Y}$ & $\mathrm{Y}$ & $\mathrm{Y}$ & $\mathrm{Y}$ & $\mathrm{Y}$ & $\mathrm{N}$ & $\mathrm{Y}$ \\
\hline $\begin{array}{l}\text { 5. Was a sample size justification, power } \\
\text { description, or variance and effect estimates } \\
\text { provided? }\end{array}$ & $\mathrm{N}$ & $\mathrm{Y}$ & $\mathrm{N}$ & $\mathrm{N}$ & $\mathrm{N}$ & $\mathrm{N}$ & $\mathrm{Y}$ & $\mathrm{N}$ & $\mathrm{Y}$ \\
\hline $\begin{array}{l}\text { 6. For the analyses in this paper, were the } \\
\text { exposure(s) of interest measured prior to the } \\
\text { outcome(s) being measured? }\end{array}$ & $\mathrm{N}$ & $\mathrm{N}$ & $\mathrm{N}$ & $\mathrm{N}$ & $\mathrm{N}$ & $\mathrm{N}$ & $\mathrm{N}$ & $\mathrm{N}$ & $\mathrm{N}$ \\
\hline $\begin{array}{l}\text { 7. Was the timeframe sufficient so that one could } \\
\text { reasonably expect to see an association between } \\
\text { exposure and outcome if it existed? }\end{array}$ & $\mathrm{N}$ & $\mathrm{N}$ & $\mathrm{N}$ & $\mathrm{N}$ & $\mathrm{N}$ & $\mathrm{N}$ & $\mathrm{N}$ & $\mathrm{N}$ & $\mathrm{N}$ \\
\hline $\begin{array}{l}\text { 8. For exposures that can vary in amount or level, } \\
\text { did the study examine different levels of the } \\
\text { exposure as related to the outcome (e.g., } \\
\text { categories of exposure, or exposure measured as } \\
\text { continuous variable)? }\end{array}$ & NA & NA & NA & NA & NA & NA & NA & NA & NA \\
\hline
\end{tabular}


Table 2. Cont.

\begin{tabular}{|c|c|c|c|c|c|c|c|c|c|}
\hline & \multicolumn{2}{|c|}{ Hallgren et al., Hudek et al., } & $\begin{array}{l}\text { Park et al., } \\
\quad 2018\end{array}$ & $\begin{array}{l}\text { Longo et al., } \\
2009\end{array}$ & $\begin{array}{l}\text { Longo et al., } \\
\quad 2010\end{array}$ & $\begin{array}{l}\text { Longo et al., } \\
\quad 2014\end{array}$ & $\begin{array}{l}\text { Papalia et al., } \\
2011\end{array}$ & \multicolumn{2}{|c|}{ Savitskaya et al., Kim et al. } \\
\hline $\begin{array}{l}\text { 9. Were the exposure measures (independent } \\
\text { variables) clearly defined, valid, reliable, and } \\
\text { implemented consistently across all study } \\
\text { participants? }\end{array}$ & $\mathrm{Y}$ & $\mathrm{Y}$ & $\mathrm{Y}$ & $\mathrm{Y}$ & $\mathrm{Y}$ & $\mathrm{Y}$ & Y & $\mathrm{Y}$ & Y \\
\hline $\begin{array}{l}\text { 10. Was the exposure(s) assessed more than once } \\
\text { over time? }\end{array}$ & $\mathrm{N}$ & $\mathrm{N}$ & $\mathrm{N}$ & $\mathrm{N}$ & $\mathrm{N}$ & $\mathrm{N}$ & $\mathrm{N}$ & $\mathrm{N}$ & $\mathrm{N}$ \\
\hline $\begin{array}{l}\text { 12. Were the outcome assessors blinded to the } \\
\text { exposure status of participants? }\end{array}$ & NR & NR & NR & NR & NR & NR & NR & NR & NR \\
\hline $\begin{array}{l}\text { 13. Was loss to follow-up after baseline } 20 \% \text { or } \\
\text { less? }\end{array}$ & $\mathrm{Y}$ & $\mathrm{Y}$ & $\mathrm{Y}$ & $\mathrm{Y}$ & Y & $\mathrm{Y}$ & $\mathrm{Y}$ & $\mathrm{Y}$ & $\mathrm{Y}$ \\
\hline $\begin{array}{l}\text { 14. Were key potential confounding variables } \\
\text { measured and adjusted statistically for their } \\
\text { impact on the relationship between exposure(s) } \\
\text { and outcome(s)? }\end{array}$ & $\mathrm{N}$ & $\mathrm{N}$ & $\mathrm{N}$ & $\mathrm{Y}$ & $\mathrm{N}$ & $\mathrm{N}$ & Y & $\mathrm{N}$ & $\mathrm{Y}$ \\
\hline Number of "yes" & 7 & 8 & 6 & 8 & 6 & 6 & 9 & 5 & 9 \\
\hline
\end{tabular}

Y, yes; N, no; CD, cannot determine; NA, not applicable; NR, not reported. 


\section{Discussion}

Results of RCD immunologic biomarker studies through biopsy show significant increases in pro-inflammatory markers cyclooxygenase COX-1 and COX-2, TNF $\alpha$, IL- $1 \beta$, IL-6, hypoxia-inducible factors (HIFs), VEGF, and degenerative enzymes MMP-1, MMP-9, and MMP-13 in patients with RCD [16-44]. Compared with current available serological studies, significantly higher levels of IL-1 $\beta$ and VEGF [13] were also found in blood samples of the patients but not MMP-1 and/or MMP-9 [14]. The remainder of the pro-inflammatory markers are pending further investigation.

Importantly, Savitskaya et al. (2011) observed that changes in serum levels of IL-1 $\beta$, IL-8, VEGF, ANG, and IL-10 are statistically significant and specific in distinguishing between patients with RCD and control subjects [13]. As stated, levels of IL-1 $\beta$ and VEGF are demonstrated to be significantly higher in patients with RCD through both biopsy and serum samples; this may indicate that the inflammatory process is involved in RCD locally and systemically. Interleukin- $1 \beta$ is a cytokine serving as an inflammatory mediator which induces the expressions of MMP-1, MMP-3, MMP-13, IL-1, IL-6, and COX-2 in tendon cells. It functions for the modulation of immune responses and tissue remodelling $[41,45]$. Significant correlations were also computed between overexpression of VEGF and clinical presentations of advanced stages of $\operatorname{RCD}(\mathrm{r}=0.75 ; p<0.01)$, average microvascular density $(\mathrm{r}=0.68, p<0.01)$, and visual analog scores $(\mathrm{r}=0.75, p<0.01)$. VEGF is a pro-inflammatory substance and signal protein that is produced by endothelial and mesothelial cells which can be found in blood vessels and internal organs [46-48]. It plays an important role in angiogenesis for migration and mitosis of endothelial cells, increasing matrix metalloproteinase activities, stimulating vessels to invade hyper-vascularized tissue, and producing fenestrations in endothelial cells $[16,49,50]$. Therefore, serum VEGF may serve as a potential biomarker for measuring treatment responsiveness and for monitoring the severity of RCD pending further longitudinal study.

Hypo-HDLemia was found to have a significant odds ratio of 2.17 when comparing patients with RCD to the control group [51]. This finding adds to the existing risk factors for RCD. In the study by Hudek et al. (2019), several fatty acids levels were found to be significantly higher in patients with RCD but the Omega-3 Index in the RCD group was significantly lower than that in the control group $(5.01 \pm 1.27$ verses $6.01 \pm 1.39$, $p=0.03$ [ [52]. These results align with a recent systematic review that showed an association between metabolic syndrome and RCD [8]. In Kim et al.'s (2021) study, significantly lower levels of vitamin B12, vitamin D, and phosphorus were found in serum samples of patients with RCD compared with control group patients [53]. In addition, the higher vitamin D levels were calculated to have a significantly lower odds ratio $(0.89,95 \%$ CI 08.2-0.96, $p=0.006)$ for degenerative RC tears. Summarizing the results from Savitskaya et al. (2011), Park et al. (2018), Hudek et al. (2019), and Kim et al. (2021), we propose the hypothesis that the pathological mechanism of RCD may involve systemic inflammatory components. These substances correlate with diverse metabolic factors such as body composition, age, diet, and exercise habits. Further investigation is required in order to determine the exact pathological mechanism between these substances and RCD as well as their usefulness as specific indicators for detecting or monitoring RCD.

A lower HDL level was statistically correlated with $\mathrm{RCD}$ (odds ratio $=0.99$, $p=0.035$ ) [51] but its clinical relevance remains to be determined. Similarly, higher NPN was found in patients with RCD than with those in the control group [54] but the difference $(37.2 \pm 8.9 \mathrm{mg} / \mathrm{dL}$ versus $35.9 \pm 10.2 \mathrm{mg} / \mathrm{dL}$, effect size $=0.14$ Cohen's d) may be too small to draw a clinical conclusion from. Although Longo et al. (2009) reported significantly higher fasting plasma glucose levels in the RCD group, values were within the normoglycemic range [55]. Furthermore, the fasting serum glucose level of patients with RCD reported in Kim et al.'s (2021) study was rather insignificant [56]. Other findings were that the levels of biomarkers MMP-1,2,3,7,9, TIMP-2,3,4, LDL, TG, non-HDL, triglycerides, cholesterol, fibrinogen, and creatinine were similar among RCD and control group patients. 
Although several recent studies did not meet the inclusion criteria of this systematic review as a result of their methodologies, their results provide important insights. Hedderson et al. (2020) investigated plasma samples of patients with acute $(<48 \mathrm{~h})$ shoulder muscle injuries not specific to RC. In contrast to Savitskaya et al.'s (2011) study which reported significantly lower levels of serum IL-10 in patients with RCD, significantly higher levels of plasma IL-6 and IL-10 were reported in Hedderson et al.'s (2020) study [13,57]. Their results indicate that the pathology of RCD differs from that of acute inflammation. Lee et al. (2020) conducted research comparing two groups of patients, those who had revision surgery within 2 years after the first repair, and who did not require additional surgery during the same period. Their results showed significantly higher levels of serum total cholesterol $(210.2 \pm 40.0$ versus $189.7 \pm 39.1, p=0.012)$ and low-density lipoprotein $(130.7 \pm 28.7$ versus $115.5 \pm 26.9, p=0.008)$ in the group with revision surgery [58]. The findings are different from Park et al.'s (2018) study which reported no significant differences between the serum total cholesterol levels in patients with RCD and those in the control group (200 \pm 35 versus $198 \pm 37, p>0.05$ ) [51]. Therefore, even serum cholesterol level may not be specific for detecting RCD but could be an indicator for planning RCD treatments. Similarly, Suh et al. (2020) conducted a research study to evaluate serum levels of high-sensitive C-reactive protein and high-density lipoprotein in patients with RCD. However, their inclusion was specific to patients with RCD and hand osteoarthritis, and which condition the results were due to was uncertain [59].

In addition to the inflammatory markers and biochemical substances mentioned in this review, a recent study has shown the presence of estrogen and progesterone receptors in supraspinatus tendon biopsies of patients with RCD [60]. Therefore, sex hormones can also be potential markers to detect physiological changes in RCD since estrogen and testosterone have been proposed to play important roles in tendon collagen synthesis and turnover, respectively [61].

Serological assessment for biomarkers is convenient and less invasive than biopsy studies; however, techniques based on Enzyme-Linked Immunosorbent Assays (ELISA) may only be available in specialized laboratories, often at high costs. The use of biosensors such as electrochemical, optical, quartz crystal microbalance, and wearable biosensors can provide more rapid, non-invasive ways to detect nucleic acids, enzymes, antibodies, and peptides [62]. Such techniques are available for osteoarthritis studies, thus they may also be applicable for RCD investigations in addition to studies for other musculoskeletal diseases.

\subsection{Limitations}

Overall, the number of serological studies included in this review is less than the previous review on biopsy studies [5,6]. For article selection, automated filters were used; relevant articles may have been excluded if they were not properly classified. The studies included in this systematic review are all cross-sectional studies without repetitive longitudinal assessment; therefore, it remains unclear if levels of biomarkers change after treatment to reflect responsiveness. Certain risks of bias may exist based on the quality of the methodology. All included studies are level III evidence, yet the overall level of evidence is low. Meta-analysis was not conducted since the included studies do not share similar outcome measures.

In most of the included studies diagnosis of RCD was confirmed by MRI, but in the study conducted by Hallgren et al. (2012), diagnosis was verified by sonographic examination [14]. Furthermore, Papalia et al. (2011) did not specify the diagnostic imaging means used for subject inclusion in their study [54]. Some of the studies did not indicate specifically whether subjects with RC tears were symptomatic or asymptomatic; for example, the study group used by Park et al. (2018) consisted of patients with both symptomatic and asymptomatic RC tears [51]. Patients with comorbidities were excluded from this systematic review, but in actual situations patients with RCD around age 40-60 may have other comorbidities such as osteoarthritis or diabetes mellitus. 


\title{
4.2. Future Research
}

As mentioned, several immune biomarkers were found to be elevated in the biopsy samples of RCD patients including COX-1, COX-2, TNF $\alpha$, and HIFs, and should be considered for further serological studies. Additional longitudinal research on blood biomarkers including VEGF, ANG, IL-1 $\beta$, IL-8, and IL-10 may determine their relevance as suitable and sensitive biomarkers in monitoring RCD disease status.

\section{Conclusions}

This is the first systematic review to summarize the case-control and cohort studies of serological investigations in RCD patients. The results of the included studies demonstrate significant changes in the levels of some biochemical substances in blood samples of RCD patients. The findings indicate systemic changes occurring in patients with RCD, and the review's results provide new insights in understanding the complex pathology of RCD. Secondly, the results suggest serological tests for certain biochemical substances can be possibly applied clinically to monitor the status of RCD, which could be helpful in management plans. Further longitudinal studies are expected to evaluate further the usefulness and sensitivity of serological tests for RCD.

Author Contributions: Conceptualization, C.N.L., B.P.L.L. and S.P.C.N.; methodology, C.N.L., B.P.L.L. and S.P.C.N.; validation, B.P.L.L. and S.P.C.N.; investigation, C.N.L., B.P.L.L. and S.P.C.N.; writing - original draft preparation, C.N.L., B.P.L.L. and S.P.C.N.; writing-review and editing, C.N.L., B.P.L.L. and S.P.C.N.; visualization, C.N.L.; supervision, B.P.L.L. and S.P.C.N.; project administration, C.N.L. All authors have read and agreed to the published version of the manuscript.

Funding: This research was supported by the Hong Kong Polytechnic University (Project ID: P0033831/ZVRR) to Ngai SPC.

Institutional Review Board Statement: Not applicable.

Informed Consent Statement: Not applicable.

Conflicts of Interest: The authors declare no conflict of interest.

\section{Appendix A}

\author{
Appendix A.1. Search String on PubMed \\ (((shoulder) OR ("rotator cuff"[Mesh])) AND (((((pain) OR ("disease"[Mesh])) OR (im- \\ pingement)) OR (tear)) OR (tend*))) AND (((blood) OR ("serum"[Mesh])) OR \\ ("plasma"[Mesh])). \\ Appendix A.2. Search String on Virtual Health Library \\ (((shoulder) OR (rotator cuff)) AND (((((pain) OR (disease)) OR (impingement)) OR \\ (tear)) OR (tend*))) AND (((blood) OR (serum)) OR (plasma)).
}

\section{References}

1. Dang, A.; Davies, M. Rotator Cuff Disease: Treatment Options and Considerations. Sports Med. Arthrosc. 2018, 26, 129-133. [CrossRef] [PubMed]

2. Whittle, S.; Buchbinder, R. Rotator Cuff Disease. Ann. Intern. Med. 2015, 162, ITC1-ITC16. [CrossRef] [PubMed]

3. Thorpe, A.; Hurworth, M.; O'Sullivan, P.; Mitchell, T.; Smith, A. Rising trends in surgery for rotator cuff disease in Western Australia. ANZ J. Surg. 2016, 86, 801-804. [CrossRef] [PubMed]

4. Schairer, W.W.; Nwachukwu, B.U.; Fu, M.C.; Warren, R.F. Risk Factors for Short-term Complications After Rotator Cuff Repair in the United States. Arthrosc. J. Arthrosc. Relat. Surg. 2018, 34, 1158-1163. [CrossRef] [PubMed]

5. Dean, B.J.F.; Franklin, S.L.; Carr, A.J. A systematic review of the histological and molecular changes in rotator cuff disease. Bone Jt. Res. 2013, 1, 158-166. [CrossRef] [PubMed]

6. Morita, W.; Dakin, S.G.; Snelling, S.J.B.; Carr, A.J. Cytokines in tendon disease. Bone Jt. Res. 2017, 6, 656-664. [CrossRef]

7. Notarnicola, A.; Maccagnano, G.; Maresca, L.; Oliva, M.C.; Fari, G.; Papagni, G.; Pignatelli, G.; Covelli, I.; Gioia, G.; Bianchi, F.P.; et al. Is extracorporeal shockwave therapy effective even in the treatment of partial rotator cuff tear? J. Biol. Regul. Homeost. Agents 2020, 34, 709-714. [CrossRef] 
8. Burne, G.; Mansfield, M.; Gaida, J.E.; Lewis, J.S. Is there an association between metabolic syndrome and rotator cuff-related shoulder pain? A systematic review. BMJ Open Sport Exerc. Med. 2019, 5, e000544. [CrossRef]

9. Higgins, J.P.T.; Thomas, J.; Chandler, J.; Cumpston, M.; Li, T.; Page, M.J.; Welch, V.A. Cochrane Handbook for Systematic Reviews of Interventions. Version 6.2 (updated February 2021); Cochrane. 2019. Available online: https://training.cochrane.org/ handbook/current (accessed on 2 November 2021).

10. Page, M.J.; McKenzie, J.E.; Bossuyt, P.M.; Boutron, I.; Hoffmann, T.C.; Mulrow, C.D.; Shamseer, L.; Tetzlaff, J.M.; Moher, D. Updating guidance for reporting systematic reviews: Development of the PRISMA 2020 statement. J. Clin. Epidemiol. 2021, 134, 103-112. [CrossRef]

11. Longo, U.G.; Margiotti, K.; Petrillo, S.; Rizzello, G.; Fusilli, C.; Maffulli, N.; De Luca, A.; Denaro, V. Genetics of rotator cuff tears: No association of col5a1 gene in a case-control study. BMC Med. Genet. 2018, 19, 217. [CrossRef]

12. Peach, C.A.; Zhang, Y.; Dunford, J.E.; Brown, M.A.; Carr, A.J. Cuff tear arthropathy: Evidence of functional variation in pyrophosphate metabolism genes. Clin. Orthop. Relat. Res. 2007, 462, 67-72. [CrossRef] [PubMed]

13. Savitskaya, Y.A.; Izaguirre, A.; Sierra, L.; Perez, F.; Cruz, F.; Villalobos, E.; Almazan, A.; Ibarra, C. Effect of Angiogenesis-Related Cytokines on Rotator Cuff Disease: The Search for Sensitive Biomarkers of Early Tendon Degeneration. Clin. Med. Insights Arthritis Musculoskelet. Disord. 2011, 4, CMAMD.S7071. [CrossRef] [PubMed]

14. Hallgren, H.C.B.; Eliasson, P.; Aspenberg, P.; Adolfsson, L.E. Elevated plasma levels of TIMP-1 in patients with rotator cuff tear. Acta Orthop. 2012, 83, 523-528. [CrossRef] [PubMed]

15. Burns, P.B.; Rohrich, R.J.; Chung, K.C. The Levels of Evidence and Their Role in Evidence-Based Medicine. Plast. Reconstr. Surg. 2011, 128, 305-310. [CrossRef] [PubMed]

16. Lakemeier, S.; Reichelt, J.J.; Patzer, T.; Fuchs-Winkelmann, S.; Paletta, J.R.; Schofer, M.D. The association between retraction of the torn rotator cuff and increasing expression of hypoxia inducible factor $1 \alpha$ and vascular endothelial growth factor expression: An immunohistological study. BMC Musculoskelet. Disord. 2010, 11, 230. [CrossRef]

17. Lakemeier, S.; Reichelt, J.J.; Timmesfeld, N.; Fuchs-Winkelmann, S.; Paletta, J.R.; Schofer, M.D. The relevance of long head biceps degeneration in the presence of rotator cuff tears. BMC Musculoskelet. Disord. 2010, 11, 191. [CrossRef]

18. Castagna, A.; Cesari, E.; Gigante, A.; Conti, M.; Garofalo, R. Metalloproteases and their inhibitors are altered in both torn and intact rotator cuff tendons. Musculoskelet. Surg. 2013, 97, 39-47. [CrossRef]

19. Shindle, M.K.; Chen, C.C.T.; Robertson, C.; DiTullio, A.E.; Paulus, M.C.; Clinton, C.M.; Cordasco, F.A.; Rodeo, S.A.; Warren, R.F Full-thickness supraspinatus tears are associated with more synovial inflammation and tissue degeneration than partial-thickness tears. J. Shoulder Elb. Surg. 2011, 20, 917-927. [CrossRef]

20. Chaudhury, S.; Xia, Z.; Thakkar, D.; Hakimi, O.; Carr, A.J. Gene expression profiles of changes underlying different-sized human rotator cuff tendon tears. J. Shoulder Elb. Surg. 2016, 25, 1561-1570. [CrossRef]

21. Jacob, J.; Eisemon, E.; Sheibani-Rad, S.; Patel, A.; Jacob, T.; Houeka, J. Matrix Metalloproteinase Levels as a Marker for Rotator Cuff Tears. Orthopedics 2012, 35, e474-e478. [CrossRef]

22. Lehmann, L.; Schollmeyer, A.; Stoeve, J.; Scharf, H.-P. Biochemische Analyse der Synovialflüssigkeit bei Patienten mit und ohne Rotatorenmanschettendefekt. Z. Orthop. Unfall. 2009, 148, 90-94. [CrossRef] [PubMed]

23. Lo, I.K.Y.; Marchuk, L.L.; Hollinshead, R.; Hart, D.A.; Frank, C.B. Matrix Metalloproteinase and Tissue Inhibitor of Matrix Metalloproteinase mRNA Levels are Specifically Altered in Torn Rotator Cuff Tendons. Am. J. Sports Med. 2004, 32, 1223-1229. [CrossRef] [PubMed]

24. Blaine, T.A.; Cote, M.A.; Proto, A.; Mulcahey, M.; Lee, F.Y.; Bigliani, L.U. Interleukin- $\beta \beta$ stimulates stromal-derived factor- $1 \alpha$ expression in human subacromial bursa. J. Orthop. Res. 2011, 29, 1695-1699. [CrossRef] [PubMed]

25. Gotoh, M.; Hamada, K.; Yamakawa, H.; Nakamura, M.; Yamazaki, H.; Ueyama, Y.; Tamaoki, N.; Inoue, A.; Fukuda, H. Perforation of rotator cuff increases interleukin 1beta production in the synovium of glenohumeral joint in rotator cuff diseases. J. Rheumatol. 2000, 27, 2886-2892.

26. Gotoh, M.; Hamada, K.; Yamakawa, H.; Yanagisawa, K.; Nakamura, M.; Yamazaki, H.; Inoue, A.; Fukuda, H. Interleukin-1induced glenohumeral synovitis and shoulder pain in rotator cuff diseases. J. Orthop. Res. 2002, 20, 1365-1371. [CrossRef]

27. Ko, J.; Wang, F.; Huang, H.; Wang, C.; Tseng, S.; Hsu, C. Increased IL-1 $\beta$ expression and myofibroblast recruitment in subacromial bursa is associated with rotator cuff lesions with shoulder stiffness. J. Orthop. Res. 2008, 26, 1090-1097. [CrossRef]

28. Joseph, M.; Maresh, C.M.; McCarthy, M.B.; Kraemer, W.J.; Ledgard, F.; Arciero, C.L.; Anderson, J.M.; Nindl, B.C.; Mazzocca, A.D Histological and molecular analysis of the biceps tendon long head post-tenotomy. J. Orthop. Res. 2009, 27, 1379-1385. [CrossRef]

29. Yanagisawa, K.; Hamada, K.; Gotoh, M.; Tokunaga, T.; Oshika, Y.; Tomisawa, M.; Lee, Y.H.; Handa, A.; Kijima, H.; Yamazaki, H.; et al. Vascular endothelial growth factor (VEGF) expression in the subacromial bursa is increased in patients with impingement syndrome. J. Orthop. Res. 2001, 19, 448-455. [CrossRef]

30. Millar, N.L.; Wei, A.Q.; Molloy, T.J.; Bonar, F.; Murrell, G.A.C. Cytokines and apoptosis in supraspinatus tendinopathy. J. Bone Jt. Surg. Br. 2009, 91-B, 417-424. [CrossRef]

31. Millar, N.L.; Akbar, M.; Campbell, A.L.; Reilly, J.H.; Kerr, S.C.; McLean, M.; Frleta-Gilchrist, M.; Fazzi, U.G.; Leach, W.J.; Rooney, B.P.; et al. IL-17A mediates inflammatory and tissue remodelling events in early human tendinopathy. Sci. Rep. 2016, 6, 27149. [CrossRef]

32. Dakin, S.G.; Martinez, F.O.; Yapp, C.; Wells, G.; Oppermann, U.; Dean, B.J.F.; Smith, R.D.J.; Wheway, K.; Watkins, B.; Roche, L.; et al. Inflammation activation and resolution in human tendon disease. Sci. Transl. Med. 2015, 7, 311ra173. [CrossRef] 
33. Sakai, H.; Fujita, K.; Sakai, Y.; Mizuno, K. Immunolocalization of cytokines and growth factors in subacromial bursa of rotator cuff tear patients. Kobe J. Med. Sci. 2001, 47, 25-34. [PubMed]

34. Wang, M.-X.; Wei, A.; Yuan, J.; Clippe, A.; Bernard, A.; Knoops, B.; Murrell, G.A.C. Antioxidant Enzyme Peroxiredoxin 5 Is Upregulated in Degenerative Human Tendon. Biochem. Biophys. Res. Commun. 2001, 284, 667-673. [CrossRef] [PubMed]

35. Benson, R.T.; McDonnell, S.M.; Knowles, H.J.; Rees, J.L.; Carr, A.J.; Hulley, P.A. Tendinopathy and tears of the rotator cuff are associated with hypoxia and apoptosis. J. Bone Jt. Surg. Br. 2010, 92-B, 448-453. [CrossRef] [PubMed]

36. Benson, R.T.; McDonnell, S.M.; Rees, J.L.; Athanasou, N.A.; Carr, A.J. The morphological and immunocytochemical features of impingement syndrome and partial-thickness rotator-cuff tear in relation to outcome after subacromial decompression. J. Bone Jt. Surg. Br. 2009, 91-B, 119-123. [CrossRef] [PubMed]

37. Millar, N.L.; Reilly, J.H.; Kerr, S.C.; Campbell, A.L.; Little, K.J.; Leach, W.J.; Rooney, B.P.; Murrell, G.A.C.; McInnes, I.B. Hypoxia: A critical regulator of early human tendinopathy. Ann. Rheum. Dis. 2012, 71, 302-310. [CrossRef]

38. Lakemeier, S.; Braun, J.; Efe, T.; Foelsch, C.; Archontidou-Aprin, E.; Fuchs-Winkelmann, S.; Paletta, J.R.J.; Schofer, M.D. Expression of matrix metalloproteinases 1, 3, and 9 in differing extents of tendon retraction in the torn rotator cuff. Knee Surgery, Sport. Traumatol. Arthrosc. 2011, 19, 1760-1765. [CrossRef]

39. Tillander, B.; Franzén, L.; Norlin, R. Fibronectin, MMP-1 and histologic changes in rotator cuff disease. J. Orthop. Res. 2002, 20, 1358-1364. [CrossRef]

40. Riley, G.P.; Curry, V.; DeGroot, J.; van El, B.; Verzijl, N.; Hazleman, B.L.; Bank, R.A. Matrix metalloproteinase activities and their relationship with collagen remodelling in tendon pathology. Matrix Biol. 2002, 21, 185-195. [CrossRef]

41. Blaine, T.A.; Kim, Y.-S.; Voloshin, I.; Chen, D.; Murakami, K.; Chang, S.-S.; Winchester, R.; Lee, F.Y.; O’Keefe, R.J.; Bigliani, L.U. The molecular pathophysiology of subacromial bursitis in rotator cuff disease. J. Shoulder Elb. Surg. 2005, 14, S84-S89. [CrossRef]

42. Lakemeier, S.; Schwuchow, S.A.; Peterlein, C.D.; Foelsch, C.; Fuchs-Winkelmann, S.; Archontidou-Aprin, E.; Paletta, J.R.; Schofer, M.D. Expression of matrix metalloproteinases 1,3, and 9 in degenerated long head biceps tendon in the presence of rotator cuff tears: An immunohistological study. BMC Musculoskelet. Disord. 2010, 11, 271. [CrossRef]

43. Osawa, T.; Shinozaki, T.; Takagishi, K. Multivariate analysis of biochemical markers in synovial fluid from the shoulder joint for diagnosis of rotator cuff tears. Rheumatol. Int. 2005, 25, 436-441. [CrossRef]

44. Voloshin, I.; Gelinas, J.; Maloney, M.D.; O'Keefe, R.J.; Bigliani, L.U.; Blaine, T.A. Proinflammatory Cytokines and Metalloproteases Are Expressed in the Subacromial Bursa in Patients With Rotator Cuff Disease. Arthrosc. J. Arthrosc. Relat. Surg. 2005, 21, 1076.e1-1076.e9. [CrossRef] [PubMed]

45. Chaudhury, S.; Carr, A.J. Lessons we can learn from gene expression patterns in rotator cuff tears and tendinopathies. J. Shoulder Elb. Surg. 2012, 21, 191-199. [CrossRef] [PubMed]

46. Mandl-Weber, S.; Cohen, C.D.; Haslinger, B.; Kretzler, M.; Sitter, T. Vascular endothelial growth factor production and regulation in human peritoneal mesothelial cells. Kidney Int. 2002, 61, 570-578. [CrossRef] [PubMed]

47. Rees, J.D.; Stride, M.; Scott, A. Tendons-Time to revisit inflammation. Br. J. Sports Med. 2014, 48, 1553-1557. [CrossRef]

48. Tang, C.; Chen, Y.; Huang, J.; Zhao, K.; Chen, X.; Yin, Z.; Heng, B.C.; Chen, W.; Shen, W. The roles of inflammatory mediators and immunocytes in tendinopathy. J. Orthop. Transl. 2018, 14, 23-33. [CrossRef]

49. Hoeben, A.; Landuyt, B.; Highley, M.S.; Wildiers, H.; Van Oosterom, A.T.; De Bruijn, E.A. Vascular Endothelial Growth Factor and Angiogenesis. Pharmacol. Rev. 2004, 56, 549-580. [CrossRef]

50. Ucuzian, A.A.; Gassman, A.A.; East, A.T.; Greisler, H.P. Molecular Mediators of Angiogenesis. J. Burn Care Res. 2010, 31, 158-175. [CrossRef]

51. Park, H.B.; Gwark, J.-Y.; Im, J.-H.; Jung, J.; Na, J.-B.; Yoon, C.H. Factors Associated with Atraumatic Posterosuperior Rotator Cuff Tears. J. Bone Jt. Surg. Am. 2018, 100, 1397-1405. [CrossRef]

52. Hudek, R.; von Schacky, C.; Passow, A.; Abdelkawi, A.F.; Werner, B.; Gohlke, F. Degenerative rotator cuff tears are associated with a low Omega-3 Index. Prostaglandins Leukot. Essent. Fatty Acids 2019, 148, 35-40. [CrossRef]

53. Kim, J.H.; Kim, G.-T.; Yoon, S.; Lee, H.I.; Ko, K.R.; Lee, S.-C.; Kim, D.K.; Shin, J.; Lee, S.-Y.; Lee, S. Low serum vitamin B12 levels are associated with degenerative rotator cuff tear. BMC Musculoskelet. Disord. 2021, 22, 364. [CrossRef]

54. Papalia, R.; Del Buono, A.; Leonardi, F.; Osti, L.; Maffulli, N.; Denaro, V. Creatinine and nonprotein nitrogen plasma levels: Possible etiopathogenetic factors in rotator cuff tears. Phys. Sportsmed. 2011, 39, 127-132. [CrossRef] [PubMed]

55. Longo, U.G.; Franceschi, F.; Ruzzini, L.; Spiezia, F.; Maffulli, N.; Denaro, V. Higher fasting plasma glucose levels within the normoglycaemic range and rotator cuff tears. Br. J. Sports Med. 2009, 43, 284-287. [CrossRef]

56. Grusky, A.Z.; Song, A.; Kim, P.; Ayers, G.D.; Higgins, L.D.; Kuhn, J.E.; Baumgarten, K.M.; Matzkin, E.; Jain, N.B. Factors Associated With Symptomatic Rotator Cuff Tears. Am. J. Phys. Med. Rehabil. 2021, 100, 331-336. [CrossRef]

57. Hedderson, W.C.; Borsa, P.A.; Fillingim, R.B.; Coombes, S.A.; Hass, C.J.; George, S.Z. Plasma Concentrations of Select Inflammatory Cytokines Predicts Pain Intensity 48 Hours Post-Shoulder Muscle Injury. Clin. J. Pain 2020, 36, 775-781. [CrossRef] [PubMed]

58. Lee, S.; Park, I.; Lee, H.A.; Shin, S.-J. Factors Related to Symptomatic Failed Rotator Cuff Repair Leading to Revision Surgeries After Primary Arthroscopic Surgery. Arthrosc. J. Arthrosc. Relat. Surg. 2020, 36, 2080-2088. [CrossRef] [PubMed]

59. Suh, Y.S.; Kim, H.-O.; Cheon, Y.-H.; Kim, M.; Kim, R.-B.; Park, K.-S.; Park, H.B.; Na, J.-B.; Moon, J.I.; Lee, S.-I. Metabolic and inflammatory links to rotator cuff tear in hand osteoarthritis: A cross sectional study. PLoS ONE 2020, 15, e0228779. [CrossRef] [PubMed] 
60. Longo, U.G.; Mazzola, A.; Carotti, S.; Francesconi, M.; Catapano, S.; Magrì, F.; Perrone, G.; Morini, S.; De Salvatore, S.; Denaro, $\mathrm{V}$. The role of estrogen and progesterone receptors in the rotator cuff disease: A retrospective cohort study. BMC Musculoskelet. Disord. 2021, 22, 891. [CrossRef]

61. Hansen, M.; Kjaer, M. Sex Hormones and Tendon. In Metabolic Influences on Risk for Tendon Disorders. Advances in Experimental Medicine and Biology; Ackermann, P., Hart, D., Eds.; Springer: Cham, Switzerland, 2016; Volume 920, pp. 139-149. [CrossRef]

62. Longo, U.G.; Candela, V.; Berton, A.; De Salvatore, S.; Fioravanti, S.; Giannone, L.; Marchetti, A.; De Marinis, M.G.; Denaro, V. Biosensors for Detection of Biochemical Markers Relevant to Osteoarthritis. Biosensors 2021, 11, 31. [CrossRef] 